\title{
Thanatology in the elderly
}

\section{Introduction}

Thanatology is the science that brings together the total of people, traditions, acts and emotions of what is thought and done about the agony and death, not in vain religious texts such as the book of the Maya, the Iching, the bodol thodol and the manual on the art of dying instructed the dying about their behavior in the final moments of life, that is why the spiritual guide occupies a privileged place in the process of goodness dying preparing the individual for the "Beyond", The challenge then for health personnel is to prepare the individual to alleviate the "more here" through palliative care.

Unfortunately the twentieth century is tanatofóbico, death continues to be seen by many as the defeat of the doctor and unfortunately this moves away from reality where the doctor routinely flees from this important life event

\section{Aging is not synonymous with getting sick}

History shows that many old people who reached senile age in full enjoyment of their mental faculties have made life more remarkable, some examples are Goethe in letters, Newton in the sciences, Marco Polo in action, among many. Aging provides mental clarity, not in vain the masterpieces are, ordinarily, of old authors. Aging does not mean getting sick, nor old age equals pathology. Being old does not mean being in a terminal state and age is not a criterion to limit therapeutic interventions.

\section{Defining the terminally ill}

Is one that, based on the diagnoses and therapies available, can be reasonably estimated and with a high probability of hitting, that will die before six months.

\section{The bad news: Communication of death to the patient}

It is necessary that the doctor take time to explain this situation and possibly a single conversation is not enough, the patient often senses the seriousness of the moment and is not as disturbed as the doctor assumes

For many seriously ill human beings death is not the worst, there are states of life much worse than death itself, in which it comes to be presented as a solution, a relief to an unbearable suffering, a desired goal.

Unattended suffering affects not only the quality of life but also the quality of the death of the human being

\section{The family}

The family and friends are important in this process, each assistant will also learn to die and when they meet a dignified death, they will also die a little.

A frightened or threatened patient is a "foolish, annoying or demanding" patient who asks for company and calls the nurse all night, a patient with a patient is more manageable and less demanding for hospital staff. The doctor must have clear the behavior that suits the patient not to create confusion in the family, giving false expectations or putting them to decide on possibilities that are not feasible, because this produces feelings of guilt or suffering.
Volume 3 Issue I - 2019

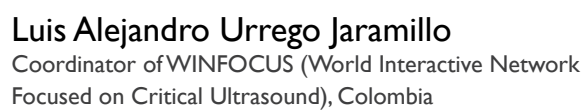

Correspondence: Luis Alejandro Urrego Jaramillo, Emergency, Critical Care Medicine and Intensive Care, ATLS Instructor American College of Surgeons, Coordinator of WINFOCUS (World Interactive Network Focused on Critical Ultrasound), Colombia, Email alejourrego@hotmail.com

Received: July 10, 2018 | Published: January 28, 2019

Respect at all times the pain of the family and understand the hostile manifestations that death can produce. The patient and the family should not be abandoned in the face of the imminence of death.

In case of conflict between the doctor and the family of the incompetent patient, the patient must be transferred to another colleague and if there is an intention to run over the patient's rights, they should always resort to justice.

\section{Listening to the patient who dies}

There are no simple formulas to give psychological support to the disease; It is basically a problem of motivation, training, an open attitude to understand the patient as a person living a process that transcends the organic.

Illness confronts us with suffering, helplessness, vulnerability and the threat of death. Illness reminds us of true human nature and awakens in all childhood fears and needs for survival. This deep meaning of the disease explains the demanding relationships that many patients establish with health professionals and their families. Also the disease generates fear of the damage of the body, to the corresponding pain and to the loss of functionality or of the aesthetic appearance, it is not superfluous to remember that the body cannot be touched without consent and without explanation, nor can it be exhibited or violated.

Another type of fear is linked to autonomy: the patient is afraid of being ignored or not consulted; subjected to treatments against his will where many times the patient submits docilely for fear of opposing the authority or the authoritarianism of the doctor for avoid being scolded, rejected and losing their care with what reacts anxiety and suffering. When the patient is making contact with his reality, he reacts with rage at the failure of his body; with rage for pain and suffering, for their fears and their real or anticipated losses. Feel anger with who has failed and has not given relief, as God or the medical staff; because the relief they offer involves pain, discipline and deprivation. The guilt also arises from failures in self-care and guilt for specific actions that are scientifically related or in the fantasies and myths of the patient, their family and their culture. The patient needs they create their pain, their discomfort, their suffering: that is real. Do not devalorize it, you have to explore in which part of the suffering is being neglected: are you afraid? Do you feel lonely? Forsaken?, Do not understand?, It produces anger and exhaustion not to be believed, especially in chronic diseases. He needs to be taken care of, given something for his illness, to give him a little time, to listen to him, we have all heard with relief "here comes the doctor". 
Cardiorespiratory resuscitation maneuvers should be discussed widely with the patient if you want resuscitation maneuvers if you need them, but when the patient's condition is terminal and the benefits are few, it can be omitted, but always judiciously evaluating the patient, his will and the family. The patient owns his life and therefore, if he freely and consciously refuses a therapy, even at the cost of his life, this determination must be respected. Sometimes a natural death certificate document is signed, in which it is ordered, that in certain circumstances artificial methods to prolong life are not used. This document must have a legal nature to be taken into account by the doctor.

\section{Pain}

The worst enemy of the patient and the greatest challenge of the doctor with the advancement of medicine ${ }^{1}$ it is possible to alleviate almost any pain. If it is intense it should be treated with powerful analgesics, even deep sedation, if the therapy shortens life cannot be said to be an act of suicide or euthanasia, it is simply a treatment that seeks to relieve pain and may have side effects such as depression Respiratory and death, effects not sought or desired with therapy. Pain should be alleviated whatever the patient's condition.

\section{Quality of life - quality of death}

Quality of life depends on subjective factors that are very difficult to measure or evaluate. It should not be confused with the concept of value of life, because if quality is variable, the value of human life is not and will always be independent of the circumstances. The quality of life must be judged as a value of the person; judiciously evaluate if there is joy of life, if satisfactions are more than frustrations and if in spite of suffering, there is a desire to live. The doctor and the health personnel should assume as their responsibility to offer good quality of death. A good death is a goal, a challenge, and includes tasks such as ensuring that the dignity of the human being is not lost at any time and always respect the patient's right to know the available options and actively participate in decisions taken about its end, thus retaining a valuable portion of control over his life. In other words, death with dignity is what someone would choose for himself if he had the opportunity to do it.

\section{Agony}

Agony, that moment that precedes death it is a reason for awe and reflection, for tears, for silences and farewells. Decision-making and profound changes the experience of the dying: When approaching death the dying person suffers physiological, psychological and spiritual. $^{2}$

Physiological experiences: there is loss of muscle tone, dysphasia, dysphagia, decreased gastrointestinal activity, difficulty controlling the sphincters, stasis of circulation, changes in vital signs and state of consciousness, immobility, blindness, vomiting, constipation, coldness, hypotension, compromise of the senses and pain.

Psychological Experiences: In addition to denial, anger and depression is common the distance from the world that surrounds it, the quenching of affectivity. He talks little, he rejects loved ones, he sleeps more time, he does not care about everything and everyone, he does not want to be disturbed. This is a solitary experience of introspection, reflection, revision, search of existential meaning and preparation for departure. The dying person must be reassured in the sense that he will never be abandoned.
Spiritual experiences: The spiritual is the unifying force that integrates and transcends the physical, emotional and social dimensions; enables and motivates to find a purpose and meaning for life by relating it to a higher being.

Ethical aspects in agony: Let die: There should be no therapeutic fierceness, prolong life is often prolonging the agony, therefore requires good judgment and interdisciplinary deliberation.

Medical ethics is a process that respects the patient's autonomy and abandons paternalism; it is also an alternative to active euthanasia and assisted suicide. Accept without ironies the extracorporeal experiences induced by hypoxia, the accumulation of carbon dioxide, insomnia, etc. At the same time as medical assistance, one should seek spiritual assistance. ${ }^{3}$

The care of a terminal patient can be grouped into several categories:

A. Therapeutic care as etiological drugs, Interventions such as placement of catheters, gastrostomies. Application of technologies such as respirators, pacemakers. All these can be omitted if the doctor, with him, considers that they are not justified for the general good of the patient.

B. Symptomatic care such as analgesics to remove pain, oxygen to relieve dyspnea or anticonvulsants, should be given and never denied to the patient, whatever their situation.

C. The cares that are called basic: hydration, giving shelter, surrounding the resting environment, grooming the patient, should not be omitted from a patient for any reason, are manifestations of humanity and affection.

The withdrawal of parenteral fluids or the gastric tube has been the subject of controversy If a respirator can be removed, why not a nasogastric tube? The question is of humanity; Feeding or quenching a person's thirst is an act of elementary human compassion (Curran, 1985) and rarely will a family understand that their loved one, the doctor, denies him the elementary care of the normal feeding and hydration

\section{Objectives}

a. Evaluate the medications that the patient receives, suspend those that are not essential for the quality of life at the end of existence.

b. Prescribe medications according to established protocol, usually subcutaneous or intravenous (Morphine - Metoclopramide Midazolam, etc.)

c. Suspend laboratory tests, blood, antibiotics, taking vital signs, etc.

d. Determine the non-application of RCCP and communicate it to the team.

e. Establish an adequate and timely communication with the patient, their relatives and Friends.

f. Evaluate spiritual and religious needs.

g. Establish the way to give the bad news to ensure that patients, family members, medical personnel, know and accept the care plan. What then is the role of the doctor? Be the main advisor and source of information, which should be provided with prudence, 
serenity, clarity of language and concepts, equanimity and willingness to respect the dignity and rights of people. Before the patient who has no chance of recovery, it is essential to ensure first of all that you have not omitted or omit any measure that could be useful in any way (relief of symptoms such as pain or respiratory distress, to give two examples is imperative until the last moment); but then there must be enough presence of courage, willpower and humility to accept what is inevitable, refrain from initiating the useless and suspend or accept that what is no longer justified is suspended. ${ }^{4-7}$

\section{Conclusion}

The doctor seeks consensus, cooperates with the patient and his family, satisfies the demands that are within his reach, respects personal ethics and presents alternative solutions for the patient to decide. The patient has the right to freedom, dignity, personal integrity, information to assistance and not suffers unnecessarily.

Uncontrolled pain and suffering, depression, adverse effects of other symptoms, abandonment, isolation, lack of tenderness, inadequate medical assistance oblige the patient to ask for euthanasia or help for suicide, however, ethical and human medical assistance, proportionate to personal and family circumstances, alleviates tensions and decreases the death request premature. It should provide all the comfort possible when the cure is not within our reach.,

To conclude the best treatment to our patients is met when we answer these simple questions, how would I want them to answer me? How would I wish that I will they try? What would I like them to do with me? That at the end of the day is nothing more than returning to our human side and practicing empathy. The doctor should not feel frustrated before the death of his patient. Medicine is not to make mankind immortal, but to help you mitigate suffering.

\section{Acknowledgments}

None.

\section{Conflicts of interest}

The authors declare there is no conflicts of interest.

\section{References}

1. Silver Rueda E, Leal Quevedo F, Mendoza Vega J. Towards a more humána medicine. 1st ed. Bogotá DC, Colombia; Panamerican Medical Editorial; 1997:15

2. Álvarez E Tiberio. The emergency doctor before the agony of the patient. The urgent patient, Editorial CIB; 2010:3-11.

3. Vélez Correa LA. Medical Ethics. 3rd ed. Medellín, Colombia: Editorial corporation for biological research CIB; 2003:18-306.

4. Arbeláez C, Alvares Echeveri T. Spirituality as a source of relief. IATREHIA. 1995;8:79-84.

5. Gómez-Batiste X, Borras JM, Fontanais MD, et al. Palliative care in Catalonia 1990-95. Palliative Medicine. 1992;6(4):321-327.

6. Gómez-Batiste Alentorn X, Roca Casas J, Pladevall Casellas C, et al. Home care, clinical monographs in primary care. 1991:131-149.

7. Gómez-Sancho M. Symptom control in the terminal cancer patient. ASTA Medical. 1992.

8. Sanz Ortiz J. The terminal disease in cancer. In: Doyma, editor. General medicine and cancer. Clinical manual. Barcelona: Medicina Clínica; 1991:173-190.

9. Sanz Ortiz J. Communication in palliative medicine. Medicina Clínica. 1992;98:416-418. 